\title{
Cutaneous leishmaniasis in a 12-year-old Syrian immigrant
}

\author{
Mohammad Alghounaim MD, Jeffrey Chivinski MD, Sapha Barkati MD MSc DTM\&H
}

Cite as: CMAJ 2022 January 24;194:E93-4. doi: 10.1503/cmaj.210847

A 12-year-old girl, who had immigrated from Syria 7 months prior, presented to her general practitioner with a 6-month history of nonpainful papules over her right hand that had increased in size and eventually ulcerated. Over a follow-up period of 2 months, she did not improve despite receiving topical corticosteroids, mupirocin, and oral trimethoprim-sulfamethoxazole for suspected superinfected atopic dermatitis. When we saw her in our tropical diseases clinic, she was afebrile and systemically well, but had erythematous plaques, edema, ulcerations and restricted mobility on her right hand (Figure 1A).

Giemsa staining from an ulcer scraping specimen showed numerous amastigotes. The culture was positive for Leishmania species, and polymerase chain reaction (PCR) identified Leishmania tropica. Because we were concerned about the functional mobility of the patient's hand, we prescribed systemic therapy with liposomal amphotericin B, based on local guidelines, which she tolerated well. At followup 4 months later, her lesions had resolved (Figure 1B) and joint mobility was restored.

Cutaneous leishmaniasis is a protozoan infection transmitted by the female sandfly. This tropical disease affects 700000 to 1 million new people annually. ${ }^{1}$ Nonhealing ulcers in travellers and migrants from endemic regions, such as Central and South America, the Mediterranean basin, the Middle East and Central Asia, should raise suspicion of cutaneous leishmaniasis. Lack of physician knowledge about the acquisition of cutaneous leishmaniasis, even during short stays abroad, has historically led to delayed diagnosis. ${ }^{2,3}$

Differential diagnoses include Nocardia species, mycobacteria, endemic fungi, vasculitis, neoplasia, sarcoidosis and pyoderma gangrenosum. Identification of parasites on smear, histopathology, culture or PCR confirm the diagnosis. ${ }^{4}$ The type of Leishmania species, concomitant mucosal involvement, the size, number and location of the lesions, and host immune status are important guides to individualized management. ${ }^{5}$ Treatment may be local (e.g., paromomycin preparations, cryotherapy, heat therapy) or systemic (e.g., pentavalent antimonials, miltefosine, azoles and amphotericin B). ${ }^{6}$

\section{References}

1. Leishmaniasis. Geneva: World Health Organization; 2021. Available: https:// www.who.int/news-room/fact-sheets/detail/leishmaniasis (accessed 2021 July 19).

2. Boggild AK, Caumes E, Grobusch MP, et al. Cutaneous and mucocutaneous leishmaniasis in travellers and migrants: a 20-year GeoSentinel Surveillance Network analysis. J Travel Med 2019;26:taz055.

3. Vandeputte M, van Henten S, van Griensven J, et al. Epidemiology, clinical pattern and impact of species-specific molecular diagnosis on management of leishmaniasis in Belgium, 2010-2018: a retrospective study. Travel Med Infect Dis 2020;38:101885.

4. Aronson NE, Joya CA. Cutaneous leishmaniasis: updates in diagnosis and management. Infect Dis Clin North Am 2019;33:101-17.

5. Barkati S, Ndao M, Libman M. Cutaneous leishmaniasis in the 21st century: from the laboratory to the bedside. Curr Opin Infect Dis 2019;32:419-25.

6. Aronson N, Herwaldt BL, Libman M, et al. Diagnosis and treatment of leishmaniasis: clinical practice guidelines by the Infectious Diseases Society of America (IDSA) and the American Society of Tropical Medicine and Hygiene (ASTMH). Am J Trop Med Hyg 2017;96:24-45. 
Competing interests: None declared.

This article has been peer reviewed.

The authors have obtained parental consent.

Affiliations: Division of Infectious Diseases (Alghounaim), Department of Pediatrics, The Montreal Children's Hospital, McGill University Health Centre; Department of Dermatology (Chivinski), Centre Hospitalier de l'Université de Montréal, Université de Montréal; J.D. MacLean Centre for Tropical Diseases at McGill University (Barkati); Research Institute of the McGill University Health Centre (Barkati); Division of Infectious Diseases (Barkati), Department of Medicine, McGill University Health Centre, Montréal, Que.

Content licence: This is an Open Access article distributed in accordance with the terms of the Creative Commons Attribution (CC BY-NCND 4.0) licence, which permits use, distribution and reproduction in any medium, provided that the original publication is properly cited, the use is noncommercial (i.e., research or educational use), and no modifications or adaptations are made. See: https://creativecommons.org/ licenses/by-nc-nd/4.0/

Acknowledgement: The authors would like to thank Faheel Naeem for proofreading the manuscript.

Correspondence to: Sapha Barkati, sapha.barkati2@mcgill.ca

Clinical images are chosen because they are particularly intriguing, classic or dramatic. Submissions of clear, appropriately labelled highresolution images must be accompanied by a figure caption. A brief explanation ( 300 words maximum) of the educational importance of the images with minimal references is required. The patient's written consent for publication must be obtained before submission. 